\title{
Metaproteomics Analysis of SARS-CoV-2-Infected Patient Samples Reveals Presence of Potential Coinfecting Microorganisms
}

\author{
Peter S. Thuy-Boun, ${ }^{\#}$ Subina Mehta, ${ }^{\#}$ Bjoern Gruening, Thomas McGowan, An Nguyen, \\ Andrew T. Rajczewski, James. E Johnson, Timothy J. Griffin, Dennis W. Wolan, and Pratik D. Jagtap*
}

Cite This: https://dx.doi.org/10.1021/acs.jproteome.0c00822

Read Online

S1 Supporting Information

ABSTRACT: In this Letter, we reanalyze published mass spectrometry data sets of clinical samples with a focus on determining the coinfection status of individuals infected with SARS-CoV-2 coronavirus. We demonstrate the use of ComPIL 2.0 software along with a metaproteomics workflow within the Galaxy platform to detect cohabitating potential pathogens in COVID-19 patients using mass spectrometry-based analysis. From a sample collected from gargling solutions, we detected Streptococcus pneumoniae (opportunistic and multidrug-resistant pathogen) and Lactobacillus rhamnosus (a probiotic component) along with SARS-Cov-2. We could also detect Pseudomonas sps. Bc-h from COVID-19 positive samples and Acinetobacter ursingii and Pseudomonas monteilii from COVID-19 negative samples collected from oro- and nasopharyngeal samples. We believe that the early detection and characterization of coinfections by using metaproteomics from COVID-19 patients will potentially impact the diagnosis and treatment of patients affected by SARS-CoV-2 infection.

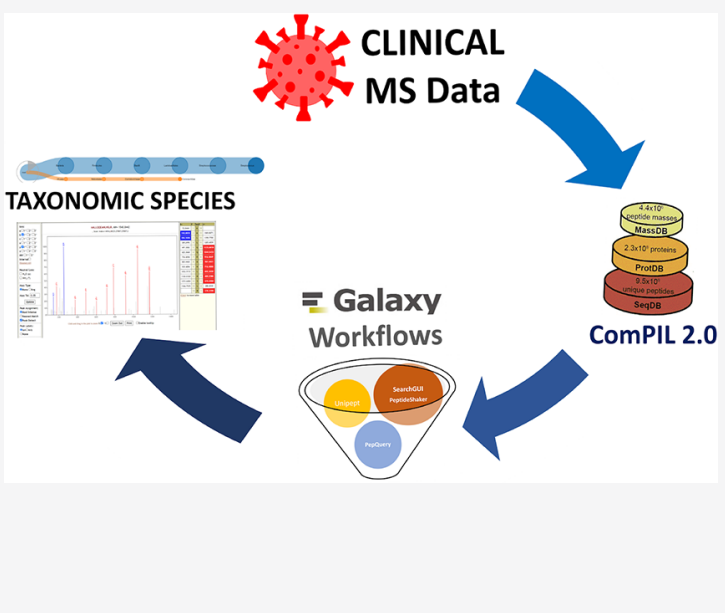

T $\mathrm{n}$ the current COVID-19 pandemic, sensitive and high1 throughput diagnostic methods are essential for the identification of SARS-CoV-2-infected individuals. Rapid and accurate tests ensure that proactive measures can be taken to trace the sources and limit the spread of the infection. The SARS-CoV-2 coronavirus can be detected from nasal swab, tracheal aspirate or blood, urine and stool specimens of infected patients by reverse transcriptase polymerase chain reaction (RT-PCR), which, along with other direct detection methods is considered the gold standard for an active infection. ${ }^{1}$ On the other hand, indirect testing methods, such as sampling for specific anti-SARS-CoV-2 antibodies, are used to infer previous exposure to the virus.

For direct detection, researchers have started exploring mass spectrometry (MS)-based assays to detect viral antigens from respiratory tract samples. ${ }^{2-4}$ High-resolution MS-based targeted assays coupled with immunoaffinity purification are being developed to improve the sensitivity and/or specificity of detection. 5 While most of these approaches detect the infection by targeting SARS-CoV-2 proteins associated with the host response, we believe that the MS-based proteomic data sets can also be further utilized to identify an understudied, but potentially important, coinfection status of the infected individual. Bacterial coinfections during respiratory-related viral outbreaks have been shown to significantly contribute to fatalities as demonstrated in both the 1918 and 2009 pandemics. $^{7,8}$ Moreover, recent research reports have also shown that many fatalities attributed to COVID-19 viral infections could be due to patients' predisposition to coinfections.

Diagnosing and managing coinfections can be complex, as it is possible that opportunistic coinfecting organisms are present in patients prior to viral infection or are acquired during hospitalization. For example, chronic bacterial infections associated with chronic obstructive pulmonary disease (COPD) can be a risk factor for patients with severe COVID-19 infection. ${ }^{10}$ Additionally, some COVID-19 patients with severe presentation are subject to prolonged mechanical ventilation augmenting their chances of developing nosocomial infections. Furthermore, the use of antibiotics to treat bacterial infections is especially high among COVID-19 patients under intensive care. The global overuse of antibiotics can lengthen the growing roster of antibiotic resistant pathogens and present problems in pairing infective organisms with appropriate antibiotics in an effective and timely fashion when using culture-based testing for coinfecting microbes. Early diagnosis of multiorganism coinfection is also necessary to avoid complications during hospitalization. Therefore, there is a

Received: October 15, 2020 


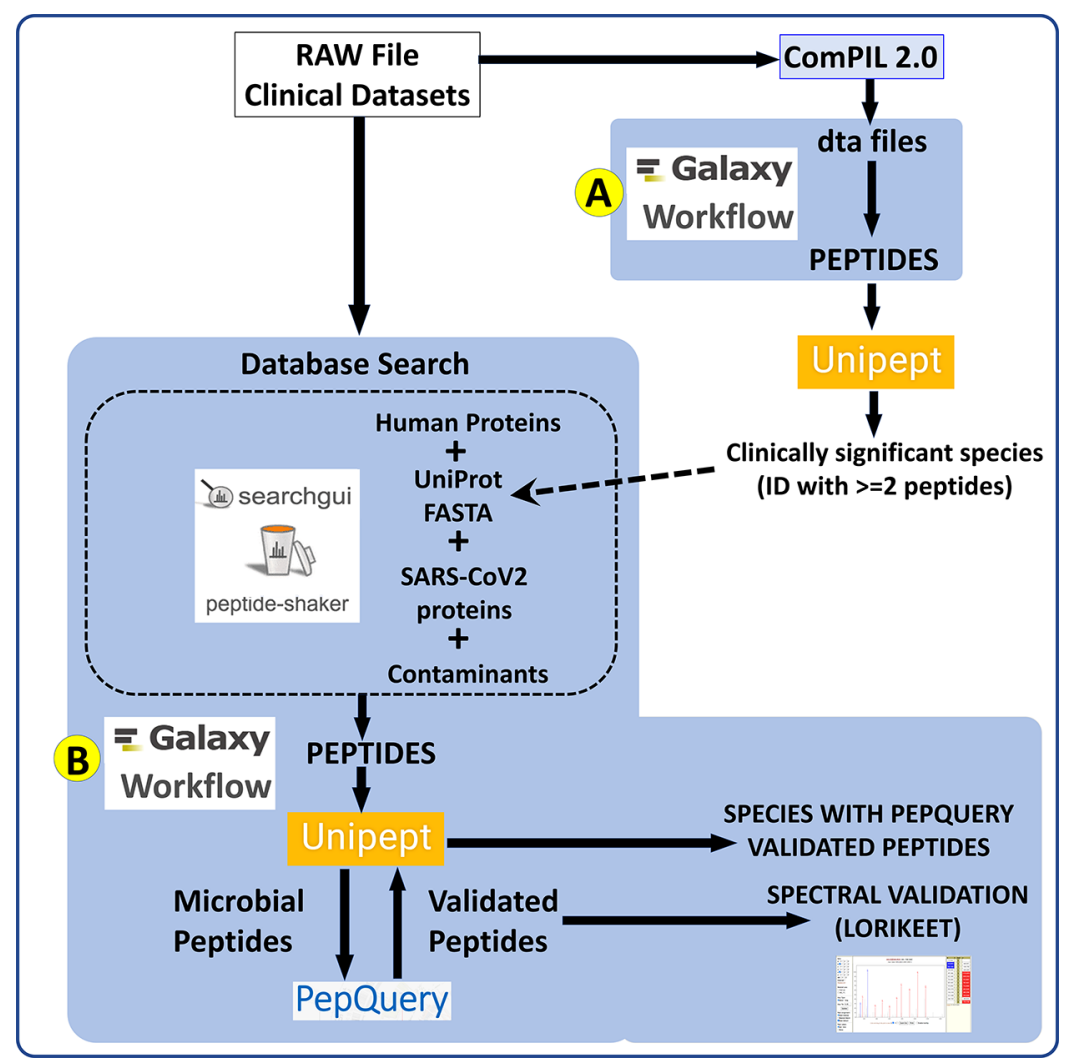

Figure 1. Metaproteomics workflows to detect cohabitating microorganisms from COVID-19 patient samples. RAW Files from clinical data sets were searched against a comprehensive UniRef database using COMPIL 2.0 software. Peptides detected from COMPIL 2.0 search were extracted using a Galaxy workflow (A) that were subjected to Unipept analysis. Clinically important species (detected with at least two peptides) were used to generate the UniProt proteins FASTA database. The RAW files were reinterrogated against a combined database of human proteins, UniProt FASTA database of detected species, SARS-CoV-2 proteins and contaminants using SearchGUI/PeptideShaker within a Metaproteomics Search and Validation Workflow (B). Confidently identified peptides were subjected to Unipept analysis to detect microbial peptides and further confirmed by using PepQuery. The confirmed peptides were used to detect species (with at least 2 peptides) after validating the spectral quality of the microbial peptides by spectral validation (Lorikeet). Species were reported to be present in a sample only when they were detected by at least two peptides in a sample or replicate.

\section{Table 1}

\begin{tabular}{|c|c|c|}
\hline data set & organisms detected in COVID-19 patient samples & link \\
\hline gargling solution $(\mathrm{PXD} 019423)^{2}$ & $\begin{array}{l}\text { Streptococcus pneumoniae, }{ }^{16,17} \text { Lactobacillus rhamnosus }{ }^{18} \text { and } \\
\text { SARS-CoV-2 }\end{array}$ & $\begin{array}{l}\text { https://covid19.galaxyproject.org/proteomics/ } \\
\text { mPXD019423/ }\end{array}$ \\
\hline $\begin{array}{l}\text { oro- and naso-pharyngeal tract } \\
(\text { PXD020394 })^{19}\end{array}$ & $\begin{array}{l}\text { Pseudomonas monteilii, }{ }^{21,22} \text { Pseudomonas sps. Bc-h, Acinetobacter } \\
\text { ursingii }{ }^{23,24} \text { and SARS-CoV-2 }\end{array}$ & $\begin{array}{l}\text { https:// covid19.galaxyproject.org/proteomics/ } \\
\text { mPXD020394/ }\end{array}$ \\
\hline respiratory tract $(\mathrm{PXD} 021328)^{20}$ & SARS-CoV-2 & $\begin{array}{l}\text { https://covid19.galaxyproject.org/proteomics/ } \\
\text { mpxd021328/ }\end{array}$ \\
\hline
\end{tabular}

need for a discovery-based analytical workflow for rapidly detecting coinfecting pathogens in COVID-19 patients, enabling subsequent characterization of antibiotic resistance and offering a roadmap for appropriate medical intervention.

In this study, we present a metaproteomic bioinformatics workflow (Figure 1) that uses MS-based data from COVID-19 patients as an input to detect peptides associated with coinfecting organisms. MS files were searched using ComPIL $2.0^{11}$ against a comprehensive protein sequence database and the detected peptides were used to find taxonomic information $^{12}$ about microorganisms present in the sample. Based on the taxonomic information, the mass spectrometry data was reinterrogated using a metaproteomics workflow (Figure 1) within the Galaxy platform to (a) match tandem mass spectra (MS/MS) against a focused custom protein sequence database of clinically significant taxa; and (b) verify detected peptides for their peptide-spectrum match (PSM) quality using the PepQuery software tool ${ }^{13}$ and the Lorikeet tandem mass spectrometry (MS/MS) spectral visualization tool. $^{14}$

We demonstrate the use of ComPIL 2.0 and metaproteomics workflow to detect cohabitating, potential pathogens in COVID-19 patients using MS-based metaproteomics analysis from three published data sets. ${ }^{2,16,18}$ We report the detection of microorganisms only when identified with at least two peptides per data set (Table 1). Each peptide detection was supported $^{15}$ by confident identification by SearchGUI/ PeptideShaker, confirmed by PepQuery analysis, and further validated by spectral annotation visualization via the Lorikeet viewer (Supplementary Data S1 and S2). From a sample collected from gargling solutions of COVID-19 positive patients, ${ }^{2}$ we detected Streptococcus pneumoniae-an oppor- 
tunistic pathogen that colonizes the mucosal surfaces of the human upper respiratory tract, ${ }^{16}$ which has been detected in COVID-19 patient samples, ${ }^{17}$ and Lactobacillus rhamnosus, which is used in oral probiotic treatment. ${ }^{18}$ We detected Pseudomonas sps. Bc-h peptides from COVID-19 positive samples, which is an unclassified strain that has not been previously shown to be associated with infection. We could detect only SARS-CoV-2 peptides from respiratory tract samples. ${ }^{19}$ We could detect Pseudomonas monteilii and Acinetobacter ursingii from COVID-19 negative samples collected from oro- and nasopharyngeal samples. ${ }^{20}$ Multidrug resistant strains of Pseudomonas monteilii have been isolated in human bronchial aspirates of hospitalized patients. ${ }^{21,22}$ Acinetobacter ursingii, a commensal bacterium present in newborns, can also cause bacteraemic infections in premature infants. ${ }^{23}$ A. ursingii has also been isolated from bronchoscopic samples of intensive-care patients. ${ }^{24}$

We believe that this untargeted metaproteomics approach offers an important step toward clinical coinfection diagnostics, wherein unbiased, direct detection of microbial peptides and inferred proteins can be performed without the requirement of accompanying metagenomic data from the patients or the use of coculturing methods. Untargeted identification of microbial peptides, followed by rigorous determination of the most reliably detected sequences, as accomplished by our bioinformatics workflow, lays the groundwork for accurate proteotyping of clinical samples ${ }^{25}$ via targeted MS-based assays. ${ }^{26}$ Moreover, the detection of coinfecting agents at the protein level can complement PCR-based methods while also offering direct evidence for an active growth of microbial agents during COVID-19 infection. We are hopeful that the workflow proposed in this study will potentially impact the diagnosis and treatment of patients affected by SARS-CoV-2 infection. Data associated with this study along with bioinformatic workflows employed are accessible via a Zenodo link, ${ }^{15}$ supplementary data (S1 and S2) and the COVID-19 Galaxy resource (https://covid19.galaxyproject.org/ proteomics/).

\section{ASSOCIATED CONTENT}

\section{Supporting Information}

The Supporting Information is available free of charge at https://pubs.acs.org/doi/10.1021/acs.jproteome.0c00822.

Supplementary S1: Methods and workflow (PDF) Supplementary S2: Spectral annotation and microbial peptides (PDF)

\section{AUTHOR INFORMATION}

\section{Corresponding Author}

Pratik D. Jagtap - University of Minnesota, Minneapolis, Minnesota 55455, United States; 10 orcid.org/0000-00030984-0973; Email: pjagtap@umn.edu

\section{Authors}

Peter S. Thuy-Boun - Scripps Research, La Jolla, California 92037, United States

Subina Mehta - University of Minnesota, Minneapolis, Minnesota 55455, United States; 10 orcid.org/0000-00019818-0537

Bjoern Gruening - Bioinformatics Group, Department of Computer Science, University of Freiburg, 79110 Freiburg im Breisgau, Germany; 이이.org/0000-0002-3079-6586
Thomas McGowan - University of Minnesota, Minneapolis, Minnesota 55455, United States

An Nguyen - University of Minnesota, Minneapolis, Minnesota 55455, United States

Andrew T. Rajczewski - University of Minnesota, Minneapolis, Minnesota 55455, United States

James. E Johnson - University of Minnesota, Minneapolis, Minnesota 55455, United States

Timothy J. Griffin - University of Minnesota, Minneapolis, Minnesota 55455, United States; 이이.org/0000-00016801-2559

Dennis W. Wolan - Scripps Research, La Jolla, California 92037, United States; (i) orcid.org/0000-0001-9879-8353

Complete contact information is available at:

https://pubs.acs.org/10.1021/acs.jproteome.0c00822

\section{Author Contributions}

\#P.S.T.-B. and S.M. contributed equally.

\section{Funding}

We acknowledge funding for this work from the grant National Cancer Institute - Informatics Technology for Cancer Research (NCI-ITCR) grant 1U24CA199347 and National Science Foundation grant 1458524. The European Galaxy server that was used for data analysis is in part funded by Collaborative Research Centre 992 Medical Epigenetics (DFG grant SFB 992/1 2012) and German Federal Ministry of Education and Research (BMBF grants 031 A538A/A538C RBC, 031L0101B/031L0101C de.NBI-epi, $031 L 0106$ de.STAIR (de.NBI)).

Notes

The authors declare no competing financial interest.

\section{REFERENCES}

(1) Russo, A.; Minichini, C.; Starace, M.; Astorri, R.; Calò, F.; Coppola, N. Vanvitelli COVID-19 group. Current Status of Laboratory Diagnosis for COVID-19: A Narrative Review. Infect. Drug Resist. 2020, 13, 2657-2665.

(2) Ihling, C.; Tänzler, D.; Hagemann, S.; Kehlen, A.; Hüttelmaier, S.; Arlt, C.; Sinz, A. Mass Spectrometric Identification of SARS-CoV2 Proteins from Gargle Solution Samples of COVID-19 Patients. J. Proteome Res. 2020, 19, 4389.

(3) Zecha, J.; Lee, C. Y.; Bayer, F. P.; Meng, C.; Grass, V.; Zerweck, J.; Schnatbaum, K.; Michler, T.; Pichlmair, A.; Ludwig, C.; Kuster, B. Data, Reagents, Assays and Merits of Proteomics for SARS-CoV-2 Research and Testing. Mol. Cell. Proteomics 2020, 19 (9), 1503-1522.

(4) Whetton, A. D.; Preston, G. W.; Abubeker, S.; Geifman, N. Proteomics and Informatics for Understanding Phases and Identifying Biomarkers in COVID-19 Disease. J. Proteome Res. 2020, 19, 4219.

(5) Gouveia, D.; Miotello, G.; Gallais, F.; Gaillard, J. C.; Debroas, S.; Bellanger, L.; Lavigne, J. P.; Sotto, A.; Grenga, L.; Pible, O.; Armengaud, J. Proteotyping SARS-CoV-2 Virus from Nasopharyngeal Swabs: A Proof-of-Concept Focused on a 3 min Mass Spectrometry Window. J. Proteome Res. 2020, 19, 4407.

(6) Renuse, S.; Vanderboom, P. M.; Maus, A. D.; Kemp, J. V.; Gurtner, K. M.; Madugundu, A. K.; Chavan, S.; Peterson, J. A.; Madden, B. J.; Mangalaparthi, K. K.; Mun, D.; Singh, S.; Kipp, B. R.; Dasari, S.; Singh, R. J.; Grebe, S. K.; Pandey, A. Development of mass spectrometry-based targeted assay for direct detection of novel SARSCoV-2 coronavirus from clinical specimens. medRxiv, Aug. 6, 2020. DOI: $10.1101 / 2020.08 .05 .20168948$.

(7) Morens, D. M.; Taubenberger, J. K.; Fauci, A. S. Predominant role of bacterial pneumonia as a cause of death in pandemic influenza: implications for pandemic influenza preparedness. J. Infect. Dis. 2008, 198 (7), 962-70. 
(8) MacIntyre, C. R.; Chughtai, A. A.; Barnes, M.; Ridda, I.; Seale, H.; Toms, R.; Heywood, A. The role of pneumonia and secondary bacterial infection in fatal and serious outcomes of pandemic influenza a(H1N1)pdm09. BMC Infect. Dis. 2018, 18 (1), 637.

(9) Zhu, X.; Ge, Y.; Wu, T.; Zhao, K.; Chen, Y.; Wu, B.; Zhu, F.; Zhu, B.; Cui, L. Co-infection with respiratory pathogens among COVID-2019 cases. Virus Res. 2020, 285, 198005.

(10) Leung, J. M.; Niikura, M.; Yang, C. W. T.; Sin, D. D. COVID19 and COPD. Eur. Respir. J. 2020, 56 (2), 2002108.

(11) Park, S. K. R.; Jung, T.; Thuy-Boun, P. S.; Wang, A. Y.; Yates, J. R.; Wolan, D. W. ComPIL 2.0: An Updated Comprehensive Metaproteomics Database. J. Proteome Res. 2019, 18 (2), 616-622.

(12) Gurdeep Singh, R.; Tanca, A.; Palomba, A.; Van der Jeugt, F.; Verschaffelt, P.; Uzzau, S.; Martens, L.; Dawyndt, P.; Mesuere, B. Mesuere B. Unipept 4.0: Functional Analysis of Metaproteome Data. J. Proteome Res. 2019, 18 (2), 606-615.

(13) Wen, B.; Wang, X.; Zhang, B. PepQuery enables fast, accurate, and convenient proteomic validation of novel genomic alterations. Genome Res. 2019, 29 (3), 485-493.

(14) Lorikeet. https://uwpr.github.io/Lorikeet/.

(15) Metaproteomics analysis of SARS-CoV-2-infected patient samples reveals presence of potential co-infecting microorganisms. Supplemental data for SARS-CoV-2 patient sample metaproteomics analysis. DOI: $10.5281 /$ zenodo.4323596.

(16) Weiser, J. N.; Ferreira, D. M.; Paton, J. C. Streptococcus pneumoniae: transmission, colonization and invasion. Nat. Rev. Microbiol. 2018, 16 (6), 355-367.

(17) Zhu, X.; Ge, Y.; Wu, T.; Zhao, K.; Chen, Y.; Wu, B.; Zhu, F.; Zhu, B.; Cui, L. Co-infection with respiratory pathogens among COVID-2019 cases. Virus Res. 2020, 285, 198005.

(18) Meurman, J. H.; Stamatova, I. Probiotics: contributions to oral health. Oral Dis. 2007, 13 (5), 443-451.

(19) Cardozo, K. H. M.; Lebkuchen, A.; Okai, G. G.; Schuch, R. A.; Viana, L. G.; Olive, A. N.; Lazari, C. D. S.; Fraga, A. M.; Granato, C. F. H.; Pintão, M. C. T.; Carvalho, V. M. Establishing a mass spectrometry-based system for rapid detection of SARS-CoV-2 in large clinical sample cohorts. Nat. Commun. 2020, 11 (1), 6201.

(20) Rivera, B.; Leyva, A.; Portela, M. M.; Moratorio, G.; Moreno, P.; Durán, R.; Lima, A. Quantitative proteomic dataset from oro- and naso-pharyngeal swabs used for COVID-19 diagnosis: Detection of viral proteins and host's biological processes altered by the infection. Data Brief 2020, 32, 106121.

(21) Aditi; Shariff, M.; Beri, K. Exacerbation of bronchiectasis by Pseudomonas monteilii: a case report. BMC Infect. Dis. 2017, 17 (1), 511.

(22) Elomari, M.; Coroler, L.; Verhille, S.; Izard, D.; Leclerc, H. Pseudomonas monteilii sp. nov., isolated from clinical specimens. Int. J. Syst. Bacteriol. 1997, 47 (3), 846-52.

(23) Yakut, N.; Kepenekli, E. K.; Karaaslan, A.; Atici, S.; Akkoc, G.; Demir, S. O.; Soysal, A.; Bakir, M. Bacteremia due to Acinetobacter ursingii in infants: Reports of two cases. Pan Afr Med. J. 2016, 23, 193.

(24) Torrego, A.; Pajares, V.; Fernández-Arias, C.; Vera, P.; Mancebo, J. Bronchoscopy in Patients with COVID-19 with Invasive Mechanical Ventilation: A Single-Center Experience. Am. J. Respir. Crit. Care Med. 2020, 202 (2), 284-287.

(25) Grenga, L.; Pible, O.; Armengaud, J. Pathogen proteotyping: A rapidly developing application of mass spectrometry to address clinical concerns. Clinical Mass Spectrometry 2019, 14, 9-17.

(26) Meyer, J. G.; Schilling, B. Clinical applications of quantitative proteomics using targeted and untargeted data-independent acquisition techniques. Expert Rev. Proteomics 2017, 14 (5), 419-429. 